\section{Penetrance of LRRK2 mutations in familial Parkinson disease}

The most common genetic cause of Parkinson disease (PD) is a mutation in the leucine-rich repeat kinase 2 (LRRK2) gene that causes a Gly2019Ser substitution, which is associated with $\sim 5-6 \%$ of familial PD cases and $1-2 \%$ of idiopathic cases. Wide variations in penetrance estimates of such mutations have been reported. Now, as part of the GenePD study, Latourelle et al. report on lifetime penetrance of LRRK2 mutations in familial PD in a large study sample.

The team studied 903 patients with PD and 58 unaffected individuals from a total of 509 families, in which at least two members had PD, plus 126 randomly selected patients with PD and 197 control individuals. All participants were screened for LRRK2 mutations; carriers of such mutations (54 individuals with Gly2019Ser mutations and 4 with Arg1441Cys mutations) were identified in 31 families, whereas no such mutations were found in the control group or healthy relatives of patients with PD.

The estimated penetrance of Gly2019Ser mutations was $67 \%$ at the maximum observed age range (90-94 years) in this sample of familial PD, compared with an estimated penetrance of $33 \%$ in randomly sampled cases from a previous study.

These results indicate that other risk factors might contribute to the onset of PD, irrespective of Gly2019Ser status. Increased penetrance in familial samples might be explained by inheritance of other susceptibility factors in family members.

Original article Latourelle JC et al. (2008) The Gly2019Ser mutation in $L R R K 2$ is not fully penetrant in familial Parkinson's disease: the GenePD study. BMC Med 6: 32

\section{Alzheimer drug worsens neurological dysfunction in multiple sclerosis}

A recent trial investigating a novel treatment for cognitive decline in patients with multiple sclerosis (MS) was cut short after some patients reported worsening of symptoms. Villoslada et al. aimed to assess whether treatment with memantine, a noncompetitive agonist of $\mathrm{N}$-methyl-D-aspartate receptors, could improve cognitive performance in patients with MS. Memantine is approved for treatment of Alzheimer disease, and has also been associated with improvements in visual disturbances (oscillopsia) in some patients with MS.

Villoslada and colleagues scheduled a 1-year, placebo-controlled, crossover study (6 months of each treatment) and planned to recruit 60 patients with MS experienced cognitive impairment. Participants were randomly assigned either placebo or memantine (30 mg per day; initial dose $10 \mathrm{mg}$ per day, increased by $10 \mathrm{mg}$ each week until $30 \mathrm{mg}$ per day was reached). The trial was halted after 19 patients had been enrolled, as some participants reported worsening of neurological symptoms, including effects on visual, cerebellar and motor function. Seven of the nine patients who reported neurological decline were in the memantine treatment group. The neurological impairment observed in the memantine group seemed to be reversible and only occurred at the maximum dose. Symptoms reverted back to baseline when the dose was reduced or the drug withdrawn completely.

While the present study indicates that $30 \mathrm{mg}$ per day memantine induces neurological impairment in patients with MS, the authors do not rule out the value of further clinical trials to understand the full effects of memantine in this group of patients.

Original article Villoslada P et al. (2008) Memantine induces reversible neurologic impairment in patients with MS. Neurology [doi:10.1212/01.wnl.0000342388.73185.80]

\section{Glioblastoma produces tumor-promoting microvesicles}

Glioblastoma cells influence their surrounding environment to promote tumor growth and stimulate angiogenesis. A study by US and Dutch researchers has shown that glioblastoma achieves this effect by releasing microvesicles containing tumor-promoting RNA and proteins.

Skog et al. cultured glioblastoma cells obtained from resected human tumors, and observed that the glioblastoma cells were covered in microvesicles $50-500 \mathrm{~nm}$ in diameter. Messenger RNAs (mRNAs) for proteins involved in angiogenesis, proliferation, immune response, cell migration and histone modification were abundant within the vesicles, 